\title{
Is General Anesthesia Needed in Endoscopic Submucosal Dissection for Lesions Located in the Mid to Upper Stomach?
}

\author{
Prasit Mahawongkajit and Jirawat Swangsri ${ }^{2}$ \\ ${ }^{1}$ Department of Surgery, Faculty of Medicine, Thammasat University, Pathumthani, ${ }^{2}$ Department of Surgery, Faculty of Medicine, Siriraj \\ Hospital, Mahidol University, Bangkok, Thailand
}

See "Clinical Outcomes and Adverse Events of Gastric Endoscopic Submucosal Dissection of the Mid to Upper Stomach under General Anesthesia and Monitored Anesthetic Care" by Chang JI, Kim TJ, Hwang NY, et al., on page 77-85. Clin Endosc 2022;55:43-44

Endoscopic submucosal dissection (ESD) is a minimally invasive and advanced procedure for treating early gastric cancer, premalignant epithelial lesions, and superficial gastrointestinal lesions. The use of ESD was first reported in Japan in the late 1990s. Since then, it has become popular worldwide. This procedure is markedly superior to endoscopic mucosal resection, as ESD can achieve adequate and en bloc removal of tumors $>20 \mathrm{~mm}$ to achieve $\mathrm{R} 0$ resection and has a lower recurrence rate. ${ }^{1}$

Despite this intrinsic superiority, ESD also requires technical proficiency and carries a risk of perforation and bleeding, especially with relatively inexperienced endoscopists. ${ }^{2}$ The incidence and volume of treatment of diseases indicated for ESD are affected by the availability of skilled endoscopists, which affects therapeutic outcomes. ${ }^{3}$

Factors, such as lesion size and location, degree of submucosal fibrosis, and submucosal invasion determine the difficulty of ESD. They can lengthen the required procedural time and result in incomplete resection and an increased risk of adverse

Received: September 29, 2021 Revised: October 6, 2021

Accepted: October 14, 2021

Correspondence: Jirawat Swangsri

12th floor, Syamindra Building, Faculty of Medicine, Siriraj Hospital, 2 Prannok Road, Bangkok 10700, Thailand

Tel: +66-2419-8013, Fax: +66-2412-9160, E-mail: jirawatmissi@gmail.com ORCID: https://orcid.org/0000-0003-3721-1859

(cc) This is an Open Access article distributed under the terms of the Creative Commons Attribution Non-Commercial License (http://creativecommons.org/ licenses/by-nc/3.0) which permits unrestricted non-commercial use, distribution, and reproduction in any medium, provided the original work is properly cited. events. ${ }^{4}$ In terms of location, the middle to upper stomach is technically challenging because of the more vascular structure, the high incidence of early gastric cancer with submucosal invasion, and the retroflexion approach in endoscopic dissection. Therefore, much research is being conducted to develop instruments and techniques to improve the effectiveness of ESD, such as the use of endoscopes and traction. ${ }^{5,6}$ However, there are limitations to their broad accessibility.

Although the gastric wall is thicker than other hollow viscous organs, ESD techniques still involve meticulous dissection and hemostasis. Combining ESD with anesthesia achieves optimal conditions for a precise procedure. However, intravenous sedation may be inadequate for a lengthy procedure, and a high volume of gastric inflation intraoperatively may cause unexpected aspiration. General anesthesia (GA) is a feasible option for high-risk situations. Therefore, in institutions with an inadequate number of experienced anesthesiologists, sedation was administered by a non-anesthesiologist as an alternative approach. In different contexts, various practices have been developed to minimize the risks presented by endoscopic-resection anesthetic methods and to improve the quality of the results. ${ }^{7,8}$

In terms of monitored anesthesia care (MAC) and ESD, Chang et al. ${ }^{9}$ first published the outcomes and adverse events of ESD of lesions in the middle to upper stomach, compared to the use of GA and MAC. Their study used a propensity score analysis based on the pathology before endoscopic treatment and the number, size, and location of gastric lesions. Similar rates were observed for en bloc resection (GA, 95.7\%; 
MAC, 97.9\%; $P=0.68$ ) and complete resection (GA, 81.9\%; MAC, $84.0 \%$; $P=0.14$ ), but with no significant difference in adverse events (GA, $16.0 \%$; MAC, $8.5 \%$; $P=0.18$ ). The overall cohort comprised all types of tumors in both the en bloc and complete resection groups, and the results were qualified according to previously published evidence. ${ }^{10}$ Logistic regression analysis indicated that both GA and MAC did not influence the outcomes or adverse events. High-volume centers with specialized endoscopists, types of endoscopes, electrosurgical knives, and instruments are essential factors and influences. Further studies are required to evaluate and confirm the findings in a prospective and large number of participants.

ESD is a challenging, advanced, and minimally invasive procedure that provides complete en bloc curative resection of superficial stomach lesions with histopathological information for proper management. The tailor-made anesthetic method for suitable patients in various contexts is ideal for our community of endoscopists. Although the outcomes of GA and MAC do not differ, minimizing gastric inflation, reducing the operating time, and achieving a proper sedation level through MAC are needed to avoid unexpected aspiration. The learning curve for this sophisticated endoscopic procedure still depends on patient volume and operator experience.

\section{Conflicts of Interest}

The authors have no potential conflicts of interest.

Funding

None.

Author Contributions

Writing-original draft: Prasit Mahawongkajit

Writing-review\&editing: Jirawat Swangsri
ORCID

Prasit Mahawongkajit

https://orcid.org/0000-0001-6144-2572

Jirawat Swangsri

https://orcid.org/0000-0003-3721-1859

\section{REFERENCES}

1. Nishizawa T, Yahagi N. Endoscopic mucosal resection and endoscopic submucosal dissection: technique and new directions. Curr Opin Gastroenterol 2017;33:315-319.

2. Misumi Y, Nonaka K. Prevention and management of complications and education in endoscopic submucosal dissection. J Clin Med 2021;10:2511.

3. Daoud DC, Suter N, Durand M, Bouin M, Faulques B, von Renteln D. Comparing outcomes for endoscopic submucosal dissection between Eastern and Western countries: a systematic review and meta-analysis. World J Gastroenterol 2018;24:2518-2536.

4. Kim JH, Nam HS, Choi CW, et al. Risk factors associated with difficult gastric endoscopic submucosal dissection: predicting difficult ESD. Surg Endosc 2017;31:1617-1626.

5. Hamada K, Horikawa Y, Shiwa Y, et al. Clinical benefit of the multibending endoscope for gastric endoscopic submucosal dissection: a randomized controlled trial. Endoscopy 2021;53:683-690.

6. Yamamoto $\mathrm{Y}$, Yahagi N, Yamamoto H, Ono H, Inoue H. Innovative therapeutic endoscopy in the upper gastrointestinal tract: review of Japan Gastroenterological Endoscopic Society core sessions. Dig Endosc 2020;32:882-887.

7. Yamashita K, Shiwaku H, Ohmiya T, et al. Efficacy and safety of endoscopic submucosal dissection under general anesthesia. World J Gastrointest Endosc 2016;8:466-471.

8. Yurtlu DA, Aslan F, Ayvat P, et al. Propofol-based sedation versus general anesthesia for endoscopic submucosal dissection. Medicine (Baltimore) 2016;95:e3680.

9. Chang JI, Kim TJ, Hwang NY, et al. Clinical outcomes and adverse events of gastric endoscopic submucosal dissection of the mid to upper stomach under general anesthesia and monitored anesthetic care. Clin Endosc 2022;55:77-85.

10. Facciorusso A, Antonino M, Di Maso M, Muscatiello N. Endoscopic submucosal dissection vs endoscopic mucosal resection for early gastric cancer: a meta-analysis. World J Gastrointest Endosc 2014;6:555-563. 